\title{
Bayesian and Semi-Bayesian Estimation of the Parameters of Generalized Inverse Weibull Distribution
}

\author{
Kamaljit Kaur \\ Panjab University, Chandigarh, India, kamaljitk010@gmail.com \\ Kalpana K. Mahajan \\ Panjab University, Chandigarh, India, kkmahajan.pu@gmail.com \\ Sangeeta Arora \\ Panjab University, Chandigarh, Inida, sarora131@gmail.com
}

Follow this and additional works at: https://digitalcommons.wayne.edu/jmasm

Part of the Applied Statistics Commons, Social and Behavioral Sciences Commons, and the Statistical Theory Commons

\section{Recommended Citation}

Kaur, Kamaljit; Mahajan, Kalpana K.; and Arora, Sangeeta (2018) "Bayesian and Semi-Bayesian Estimation of the Parameters of Generalized Inverse Weibull Distribution," Journal of Modern Applied Statistical Methods: Vol. 17 : Iss. 1, Article 22.

DOI: $10.22237 /$ jmasm/1536067915

Available at: https://digitalcommons.wayne.edu/jmasm/vol17/iss1/22

This Regular Article is brought to you for free and open access by the Open Access Journals at DigitalCommons@WayneState. It has been accepted for inclusion in Journal of Modern Applied Statistical Methods by an authorized editor of DigitalCommons@WayneState. 


\section{Bayesian and Semi-Bayesian Estimation of the Parameters of Generalized Inverse Weibull Distribution}

\section{Cover Page Footnote}

The authors are thankful to the anonymous referees and the editor for their valuable suggestions/ comments which led to considerable improvement in the manuscript. The authors are also thankful to the UGC and Purse Grant (Panjab University, Chandigarh) for providing financial support for the research work. 


\section{Bayesian and Semi-Bayesian Estimation of the Parameters of Generalized Inverse Weibull Distribution}

\author{
Kamaljit Kaur \\ Panjab University \\ Chandigarh, India
}

\author{
Kalpana K. Mahajan \\ Panjab University \\ Chandigarh, India
}

\author{
Sangeeta Arora \\ Panjab University \\ Chandigarh, India
}

Bayesian and semi-Bayesian estimators of parameters of the generalized inverse Weibull
distribution are obtained using Jeffreys' prior and informative prior under specific
assumptions of loss function. Using simulation, the relative efficiency of the proposed
estimators is obtained under different set-ups. A real life example is also given.

Keywords: Generalized inverse Weibull distribution, Bayesian, loss functions, priors

\section{Introduction}

The three-parameter Generalized Inverse Weibull distribution (GIWD), introduced by de Gusmão, Ortega, and Cordeiro (2011), is a positively skewed distribution used to model the income data and, because of its ability of possessing decreasing and unimodal failure rate, is also useful in reliability and biological studies. GIWD is the generalization of various well-known and useful distributions. Most of the sub-cases of GIWD are families of inverse distributions, which play an important role in many applications (Drapella, 1993; Jiang, Murthy, \& Ji, 2001; Nelson, 1982; Khan, Pasha, \& Pasha, 2008; Zaharim, Najid, Razali, \& Sopian, 2009) and are also fitted to income-related data. These distributions have two parameters but, in order to fit better at the tails (Lubrano, 2014; Kakwani, 1980), a distribution with three parameters (GIWD) is used in the present study.

The cdf of GIWD is

$$
\mathrm{F}(x)=\mathrm{e}^{-\gamma\left(\frac{\alpha}{x}\right)^{\beta}}, \quad x>0 ; \alpha, \beta, \gamma>0
$$

doi: 10.22237/jmasm/1536067915 | Accepted: April 27, 2017; Published: September 4, 2018.

Correspondence: Kamaljit Kaur, kamaljitk010@gmail.com 


\section{KAUR ET AL}

where $\alpha$ is scale parameter and $\beta, \gamma$ are shape parameters.

The pdf of GIWD is

$$
\mathrm{f}(x)=\gamma \beta \alpha^{\beta} x^{-(\beta+1)} \mathrm{e}^{-\gamma\left(\frac{\alpha}{x}\right)^{\beta}}
$$

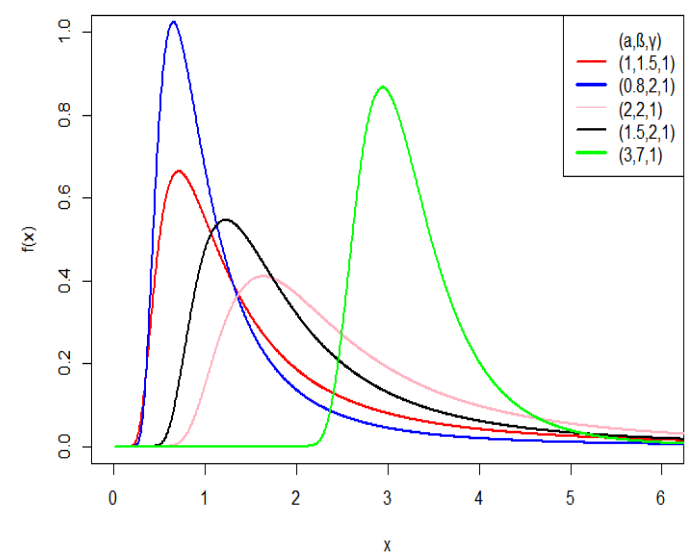

Figure 1. Plot of pdf of GIWD for various combinations of parameters

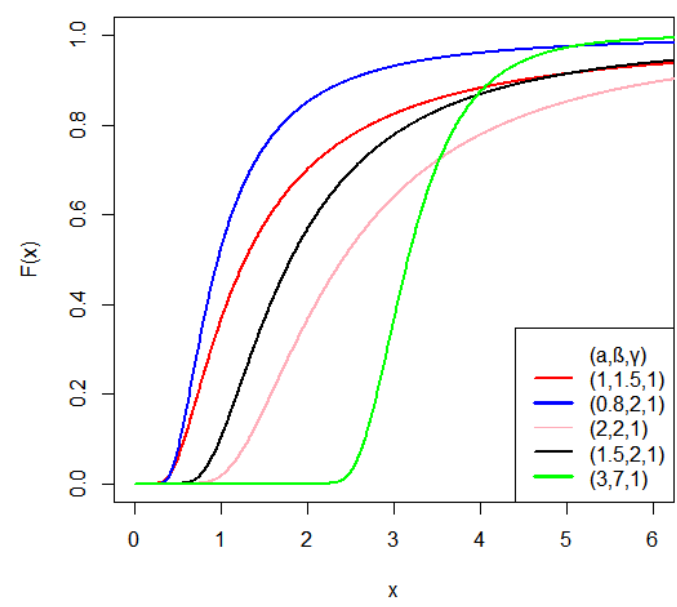

Figure 2. Plot of cdf of GIWD for various combinations of parameters 


\section{BAYESIAN ESTIMATION OF GIWD}

Sub-models:

- For $\alpha=1$, it reduces to inverse Weibull (IW) distribution.

- For $\gamma=\alpha=1$, it reduces to Fréchet (F) distribution.

- For $\beta=2, \alpha=1$, it reduces to inverse Rayleigh (IR) distribution.

- For $\gamma=\beta=1$, it reduces to inverse exponential (IE) distribution.

\section{Properties of Generalized Inverse Weibull Distribution}

The Mean and variance of GIWD have been obtained by de Gusmão et al. (2011) and are given by

$$
\gamma^{\frac{1}{\beta}} \alpha \Gamma\left(1-\beta^{-1}\right) \text { and } \gamma^{\frac{2}{\beta}} \alpha^{2}\left[\Gamma\left(1-2 \beta^{-1}\right)-\left(\Gamma\left(1-\beta^{-1}\right)\right)^{2}\right]
$$

respectively.

De Gusmão et al. (2011) have also obtained the estimators of the parameters of GIWD using a maximum likelihood method of estimation (non-Bayesian approach); they are obtained by using the likelihood function given by

$$
\mathrm{L}(x \mid \gamma, \alpha, \beta)=\gamma^{n} \beta^{n} \alpha^{n \beta}\left(\prod_{i=1}^{n} x_{i}^{-(\beta+1)}\right) \exp \left(-\gamma \alpha^{\beta} \sum_{i=1}^{n} x_{i}^{-\beta}\right)
$$

The MLEs of $\alpha$ and $\gamma$ are

$$
\begin{aligned}
& \hat{\alpha}=\left(\frac{n}{\gamma \sum_{i=1}^{n} x_{i}^{-\beta}}\right)^{\frac{1}{\beta}} \\
& \hat{\gamma}=\frac{n}{\alpha^{\beta} \sum_{i=1}^{n} x_{i}^{-\beta}}
\end{aligned}
$$

To obtain the MLE of $\beta$ equation (4) is solved using iterative numerical techniques (Newton-Raphson algorithm):

$$
\frac{n}{\beta}+n \ln \alpha-\sum_{i=1}^{n} \ln x_{i}-\gamma \alpha^{\beta} \sum_{i=1}^{n} x_{i}^{-\beta}\left(\ln \alpha-\ln \left(\sum_{i=1}^{n} x_{i}\right)\right)=0
$$




\section{KAUR ET AL}

In the classical setup, MLEs are used to estimate the parameters of GIWD. However, in the Bayesian context, estimators of these parameters still await attention of the researchers. In the Bayesian setup, one may use a full-Bayesian approach or semi-Bayesian approach, referred to as generalized maximum likelihood estimators. In the present paper, both these approaches are used to estimate the parameters of GIWD.

For Bayesian estimation, the choice of priors and loss functions are two important aspects. Both informative and non-informative priors are used for the study in case of semi-Bayesian and full-Bayesian approach. In full-Bayesian approach both symmetrical and asymmetrical loss functions are used for the study; however no loss function is required for estimating the parameters in case of semiBayesian approach.

\section{Prior and Posterior Densities}

\section{Informative Prior for the Parameters of Generalized Inverse Weibull Distribution}

The informative prior depends on the elicitation of a prior distribution based on preexisting scientific knowledge in the area of investigation. This information may be available from previous investigations or from non-statistician experts.

Assuming independence among parameters $\alpha, \beta, \gamma$ of GIWD, priors for $\alpha, \beta$, $\gamma$ are chosen to be gamma distributions as follows:

$$
\begin{aligned}
& \gamma \sim \operatorname{Gamma}\left(a_{1}, b_{1}\right) \\
& \alpha \sim \operatorname{Gamma}\left(a_{2}, b_{2}\right) \\
& \beta \sim \operatorname{Gamma}\left(a_{3}, b_{3}\right)
\end{aligned}
$$

where $\operatorname{Gamma}\left(a_{i}, b_{i}\right)$ denotes a gamma distribution given by

$$
\mathrm{f}\left(y ; a_{i}, b_{i}\right)=\frac{b_{i}^{a_{i}} y^{a_{i}-1} \exp \left(-y b_{i}\right)}{\Gamma\left(a_{i}\right)}
$$

where $y>0, a_{i}>0, b_{i}>0, i=1,2,3$, and $a_{i}$ and $b_{i}$ are the hyperparameters.

Prior density functions for $\alpha, \beta$, and $\gamma$ are 


\section{BAYESIAN ESTIMATION OF GIWD}

$$
\begin{aligned}
& \mathrm{g}\left(\alpha ; a_{2}, b_{2}\right) \propto \alpha^{a_{2}-1} \exp \left(-\alpha b_{2}\right) \\
& \mathrm{g}\left(\beta ; a_{3}, b_{3}\right) \propto \beta^{a_{3}-1} \exp \left(-\beta b_{3}\right) \\
& \mathrm{g}\left(\gamma ; a_{1}, b_{1}\right) \propto \gamma^{a_{1}-1} \exp \left(-\gamma b_{1}\right)
\end{aligned}
$$

The joint prior distribution for $\alpha, \beta$, and $\gamma$ is

$$
\mathrm{g}_{\mathrm{I}}(\alpha, \beta, \gamma) \propto \gamma^{a_{1}-1} \alpha^{a_{2}-1} \beta^{a_{3}-1} \exp \left(-\gamma b_{1}-\alpha b_{2}-\beta b_{3}\right)
$$

The joint posterior distribution for the parameters $\alpha, \beta, \gamma$ for GIWD is given by

$$
\begin{aligned}
& \mathrm{g}_{\mathrm{I}}^{*}(\alpha, \beta, \gamma \mid x) \\
& \quad=\frac{\mathrm{L}(\gamma, \alpha, \beta) \mathrm{g}\left(\gamma ; a_{1}, b_{1}\right) \mathrm{g}\left(\alpha ; a_{2}, b_{2}\right) \mathrm{g}\left(\beta ; a_{3}, b_{3}\right)}{\iiint_{0}^{\infty} \mathrm{L}(\gamma, \alpha, \beta) \mathrm{g}\left(\gamma ; a_{1}, b_{1}\right) \mathrm{g}\left(\alpha ; a_{2}, b_{2}\right) \mathrm{g}\left(\beta ; a_{3}, b_{3}\right) \partial \alpha \partial \beta \partial \gamma} \\
& \quad \propto \gamma^{n+a_{1}-1} \beta^{n+a_{3}-1} \alpha^{n \beta+a_{2}-1} \prod_{i=1}^{n} x_{i}^{-(\beta+1)} \exp \left(\left(-\gamma \alpha^{\beta} \sum_{i=1}^{n} x_{i}^{-\beta}\right)-\gamma b_{1}-\alpha b_{2}-\beta b_{3}\right)
\end{aligned}
$$

\section{Non-Informative Prior (Jeffreys' Prior) for the Parameters of Generalized Inverse Weibull Distribution}

Jeffreys' (1946) prior based on Fisher's information is defined as

$$
\pi(\theta) \propto \sqrt{\mathrm{I}(\theta)}
$$

where $\mathrm{I}(\theta)=-\mathrm{E}\left[\frac{\partial^{2}}{\partial \theta^{2}} \ln \mathrm{L}(\theta \mid x)\right]$ is Fisher's information based on likelihood function $\mathrm{L}(\theta \mid x)$.

In the case of GIWD, Jeffreys' prior is the square root of Fisher's information matrix of order $3 \times 3$, given by 


\section{KAUR ET AL}

$$
\mathrm{I}(\theta)=-\mathrm{E}\left[\begin{array}{lll}
\frac{\partial^{2}(\ln \mathrm{L}(x))}{\partial \alpha^{2}} & \frac{\partial^{2}(\ln \mathrm{L}(x))}{\partial \alpha \partial \beta} & \frac{\partial^{2}(\ln \mathrm{L}(x))}{\partial \alpha \partial \gamma} \\
\frac{\partial^{2}(\ln \mathrm{L}(x))}{\partial \beta \partial \alpha} & \frac{\partial^{2}(\ln \mathrm{L}(x))}{\partial \beta^{2}} & \frac{\partial^{2}(\ln \mathrm{L}(x))}{\partial \beta \partial \gamma} \\
\frac{\partial^{2}(\ln \mathrm{L}(x))}{\partial \gamma \partial \alpha} & \frac{\partial^{2}(\ln \mathrm{L}(x))}{\partial \gamma \partial \beta} & \frac{\partial^{2}(\ln \mathrm{L}(x))}{\partial \gamma^{2}}
\end{array}\right]
$$

where

$$
\begin{aligned}
& \frac{\partial^{2}(\ln \mathrm{L}(x))}{\partial \alpha^{2}}=-\frac{n \beta}{\alpha^{2}}-\beta(\beta-1) \gamma \alpha^{\beta-2} \sum_{i=1}^{n} x_{i}^{-\beta} \\
& \frac{\partial^{2}(\ln \mathrm{L}(x))}{\partial \beta^{2}}=-\frac{n}{\beta^{2}}-\gamma\left(\ln \alpha-\ln \left(\sum_{i=1}^{n} x_{i}\right)\right)^{2} \alpha^{\beta} \sum_{i=1}^{n} x_{i}^{-\beta} \\
& \frac{\partial^{2}(\ln \mathrm{L}(x))}{\partial \gamma^{2}}=-\frac{n}{\gamma^{2}} \\
& \frac{\partial^{2}(\ln \mathrm{L}(x))}{\partial \alpha \partial \beta}=\frac{n}{\alpha}-\gamma \alpha^{\beta-1} \sum_{i=1}^{n} x_{i}^{-\beta}\left[\beta\left(\ln \alpha-\ln \left(\sum_{i=1}^{n} x_{i}\right)\right)+1\right]=\frac{\partial^{2}(\ln \mathrm{L}(x))}{\partial \beta \partial \alpha} \\
& \frac{\partial^{2}(\ln \mathrm{L}(x))}{\partial \alpha \partial \gamma}=-\beta \alpha^{\beta-1} \sum_{i=1}^{n} x_{i}^{-\beta}=\frac{\partial^{2}(\ln \mathrm{L}(x))}{\partial \gamma \partial \alpha} \\
& \frac{\partial^{2}(\ln \mathrm{L}(x))}{\partial \beta \partial \gamma}=-\alpha^{\beta} \sum_{i=1}^{n} x_{i}^{-\beta}\left(\ln \alpha-\ln \left(\sum_{i=1}^{n} x_{i}\right)\right)=\frac{\partial^{2}(\ln \mathrm{L}(x))}{\partial \gamma \partial \beta}
\end{aligned}
$$

The expected value of double derivatives is not in a closed form, hence the explicit expression for the Jeffreys' prior is not obtained. For simplicity it is assumed that all the three parameters are independent; therefore joint prior in case of Jeffreys' prior (Guure, Ibrahimm, \& Ahmed, 2012; Singh, Singh, \& Kumar, 2011) is written as

$$
\mathrm{g}_{\mathrm{J}}(\alpha, \beta, \gamma) \propto \frac{1}{\gamma \alpha \beta}
$$




\section{BAYESIAN ESTIMATION OF GIWD}

Note that $\mathrm{g}_{\mathrm{J}}(\alpha, \beta, \gamma)$ is a special case of the informative prior by taking the hyperparameters to be zero $\left(a_{1}=b_{1}=a_{2}=b_{2}=a_{3}=b_{3}=0\right)$.

The joint posterior distribution for $\alpha, \beta, \gamma$ in the case of GIWD is given by

$$
\mathrm{g}_{\mathrm{J}}^{*}(\alpha, \beta, \gamma \mid x) \propto \gamma^{n-1} \beta^{n-1} \alpha^{n \beta-1} \prod_{i=1}^{n} x_{i}^{-(\beta+1)} \exp \left(-\gamma \alpha^{\beta} \sum_{i=1}^{n} x_{i}^{-\beta}\right)
$$

\section{Full-Bayesian Approach}

To obtain the Bayesian estimators of $\alpha, \beta$, and $\gamma$ in the case of GIWD, it may be noted that the joint posterior distributions of $\alpha, \beta$, and $\gamma$, for both informative and non-informative priors, is a ratio form that involves an integration in the denominator and cannot be reduced to a closed form. Hence the evaluation of the posterior expectation for obtaining the Bayesian estimators of $\alpha, \beta, \gamma$ will be tedious. Among the various methods suggested to approximate the ratio of integrals of the above form, the simplest one is Lindley's approximation method (Lindley, 1980), which approaches the ratio of the integrals as a whole and produces a single numerical result.

$$
\mathrm{I}(x)=\mathrm{E}\left[\mathrm{u}\left(\theta_{1}, \theta_{2}, \theta_{3}\right)\right]=\frac{\int_{\left(\theta_{1}, \theta_{2}, \theta_{3}\right)} \mathrm{u}\left(\theta_{1}, \theta_{2}, \theta_{3}\right) \mathrm{e}^{\mathrm{L}\left(\theta_{1}, \theta_{2}, \theta_{3}\right)+\mathrm{G}\left(\theta_{1}, \theta_{2}, \theta_{3}\right)} d\left(\theta_{1}, \theta_{2}, \theta_{3}\right)}{\int_{\left(\theta_{1}, \theta_{2}, \theta_{3}\right)} \mathrm{e}^{\mathrm{L}\left(\theta_{1}, \theta_{2}, \theta_{3}\right)+\mathrm{G}\left(\theta_{1}, \theta_{2}, \theta_{3}\right)} d\left(\theta_{1}, \theta_{2}, \theta_{3}\right)}
$$

where $\mathrm{L}\left(\theta_{1}, \theta_{2}, \theta_{3}\right)$ is the $\log$ of likelihood and $\mathrm{G}\left(\theta_{1}, \theta_{2}, \theta_{3}\right)$ is the $\log$ of the joint prior of $\theta_{1}, \theta_{2}$, and $\theta_{3}$.

$\mathrm{I}(x)$ can also be written as

$$
\begin{aligned}
\mathrm{I}(x)= & \mathrm{E}\left[\mathrm{u}\left(\theta_{1}, \theta_{2}, \theta_{3}\right)\right] \\
= & \mathrm{u}\left(\hat{\theta}_{1}, \hat{\theta}_{2}, \hat{\theta}_{3}\right)+\left(u_{1} a_{1}+u_{2} a_{2}+u_{3} a_{3}+a_{4}+a_{5}\right)+\frac{1}{2}\left[A \left(u_{1} \sigma_{11}+\right.\right. \\
& \left.u_{2} \sigma_{12}+u_{3} \sigma_{13}\right)+B\left(u_{1} \sigma_{21}+u_{2} \sigma_{22}+u_{3} \sigma_{23}\right)+C\left(u_{1} \sigma_{31}+u_{2} \sigma_{32}\right. \\
& \left.\left.+u_{3} \sigma_{33}\right)\right]
\end{aligned}
$$

where $\hat{\theta}_{1}, \hat{\theta}_{2}$, and $\hat{\theta}_{3}$ are the MLEs of $\theta_{1}, \theta_{2}$, and $\theta_{3}$, respectively,

$$
a_{i}=\rho_{1} \sigma_{i 1}+\rho_{2} \sigma_{i 2}+\rho_{3} \sigma_{i 3}, \quad i=1,2,3
$$




\section{KAUR ET AL}

$$
\begin{gathered}
a_{4}=u_{12} \sigma_{12}+u_{13} \sigma_{13}+u_{23} \sigma_{23} \\
a_{5}=\frac{1}{2}\left(u_{11} \sigma_{11}+u_{22} \sigma_{22}+u_{33} \sigma_{33}\right) \\
A=\sigma_{11} L_{111}+2 \sigma_{12} L_{121}+2 \sigma_{13} L_{131}+2 \sigma_{23} L_{231}+\sigma_{22} L_{221}+\sigma_{33} L_{331} \\
B=\sigma_{11} L_{112}+2 \sigma_{12} L_{122}+2 \sigma_{13} L_{132}+2 \sigma_{23} L_{232}+\sigma_{22} L_{222}+\sigma_{33} L_{332} \\
C=\sigma_{11} L_{113}+2 \sigma_{12} L_{123}+2 \sigma_{13} L_{133}+2 \sigma_{23} L_{233}+\sigma_{22} L_{223}+\sigma_{33} L_{333}
\end{gathered}
$$

subscripts $1,2,3$, on the right-hand sides above refer to $\theta_{1}, \theta_{2}$, and $\theta_{3}$, respectively, $\rho$ is the logarithm of the joint prior density,

$$
\begin{aligned}
& \rho_{i}=\frac{\partial \rho}{\partial \theta_{i}}, \quad i=1,2,3 \\
& u_{i}=\frac{\partial \mathrm{u}\left(\theta_{1}, \theta_{2}, \theta_{3}\right)}{\partial \theta_{i}}, \quad i=1,2,3 \\
& u_{i j}=\frac{\partial^{2} \mathrm{u}\left(\theta_{1}, \theta_{2}, \theta_{3}\right)}{\partial \theta_{i} \partial \theta_{j}}, \quad i, j=1,2,3 \\
& L_{i j}=\frac{\partial^{2} \mathrm{~L}\left(\theta_{1}, \theta_{2}, \theta_{3}\right)}{\partial \theta_{i} \partial \theta_{j}}, \quad i, j=1,2,3 \\
& L_{i j k}=\frac{\partial^{3} \mathrm{~L}\left(\theta_{1}, \theta_{2}, \theta_{3}\right)}{\partial \theta_{i} \partial \theta_{j} \partial \theta_{k}}, \quad i, j, k=1,2,3
\end{aligned}
$$

and $\sigma_{i j}$ is the $(i, j)^{\text {th }}$ element of the inverse of the matrix having elements $\left\{-L_{i j}\right\}$.

Remark: The expression I $(x)$ leads to the approximate expression for the Bayesian estimators.

\section{Lindley's Approximation for Generalized Inverse Weibull Distribution in case of Informative Prior}

The various terms as listed above are derived below for the three parameters $\alpha, \beta$, and $\gamma$ in the case of GIWD using informative prior. 


\section{BAYESIAN ESTIMATION OF GIWD}

The $\log$ of the joint prior density $(\rho)$ in the case of informative prior (8) is

$$
\begin{gathered}
\rho=\log (\mathrm{g}(\alpha, \beta, \gamma)) \\
=\left(a_{1}-1\right) \log \gamma+\left(a_{2}-1\right) \log \alpha+\left(a_{3}-1\right) \log \beta-b_{1} \gamma-b_{2} \alpha-b_{3} \beta \\
\rho_{1}=\frac{\partial \rho}{\partial \alpha}=\frac{a_{2}-1}{\alpha}-b_{2} \\
\rho_{2}=\frac{\partial \rho}{\partial \beta}=\frac{a_{3}-1}{\beta}-b_{3} \\
\rho_{3}=\frac{\partial \rho}{\partial \gamma}=\frac{a_{1}-1}{\gamma}-b_{1}
\end{gathered}
$$

The log-likelihood, using equation (3), is given by

$$
\begin{gathered}
\mathrm{L}(\alpha, \beta, \gamma)=\log (\mathrm{L}(x \mid \gamma, \alpha, \beta)) \\
=n \log \gamma+n \log \beta+n \beta \log \alpha-(\beta+1) \sum_{i=1}^{n} \log x_{i}-\gamma \alpha^{\beta} \sum_{i=1}^{n} x_{i}^{-\beta} \\
L_{11}=\frac{\partial^{2} \mathrm{~L}(\alpha, \beta, \gamma)}{\partial \alpha^{2}}=\frac{-n \beta}{\alpha^{2}}-\beta(\beta-1) \gamma \alpha^{\beta-2} \sum_{i=1}^{n} x_{i}^{-\beta} \\
L_{22}=\frac{\partial^{2} \mathrm{~L}(\alpha, \beta, \gamma)}{\partial \beta^{2}}=-\frac{n}{\beta^{2}}-\gamma\left(\log \alpha-\log \sum_{i=1}^{n} x_{i}\right)^{2} \alpha^{\beta} \sum_{i=1}^{n} x_{i}^{-\beta} \\
L_{12}=\frac{\partial^{2} \mathrm{~L}(\alpha, \beta, \gamma)}{\partial \alpha \partial \beta}=\frac{\partial^{2} \mathrm{~L}(\alpha, \beta, \gamma)}{\partial \gamma^{2}}=\frac{-n}{\gamma^{2}} \\
L_{13}=\frac{\partial^{2} \mathrm{~L}(\alpha, \beta, \gamma)}{\partial \alpha \partial \gamma}=-\beta \alpha_{i=1}^{n} x_{i}^{-\beta}\left[\beta\left(\log \alpha-\log \sum_{i=1}^{n} x_{i}^{-\beta}=L_{31} x_{i}\right)+1\right]=L_{21}
\end{gathered}
$$




\section{KAUR ET AL}

$$
\begin{aligned}
& L_{23}=\frac{\partial^{2} \mathrm{~L}(\alpha, \beta, \gamma)}{\partial \beta \partial \gamma}=-\alpha^{\beta} \sum_{i=1}^{n} x_{i}^{-\beta}\left(\log \alpha-\log \sum_{i=1}^{n} x_{i}\right)=L_{32} \\
& L_{111}=\frac{\partial^{3} \mathrm{~L}(\alpha, \beta, \gamma)}{\partial \alpha^{3}}=\frac{2 n \beta}{\alpha^{3}}-\gamma \beta(\beta-1)(\beta-2) \alpha^{\beta-3} \sum_{i=1}^{n} x_{i}^{-\beta} \\
& L_{222}=\frac{\partial^{3} \mathrm{~L}(\alpha, \beta, \gamma)}{\partial \beta^{3}}=-\frac{2 n}{\beta^{3}}-\gamma\left(\log \alpha-\log \sum_{i=1}^{n} x_{i}\right)^{3} \alpha^{\beta} \sum_{i=1}^{n} x_{i}^{-\beta} \\
& L_{333}=\frac{\partial^{3} \mathrm{~L}(\alpha, \beta, \gamma)}{\partial \gamma^{3}}=\frac{2 n}{\gamma^{3}} \\
& L_{113}=\frac{\partial^{3} \mathrm{~L}(\alpha, \beta, \gamma)}{\partial \alpha^{2} \partial \gamma}=-\beta(\beta-1) \alpha^{\beta-2} \sum_{i=1}^{n} x_{i}^{-\beta}=L_{131}=L_{311} \\
& L_{112}=\frac{\partial^{3} \mathrm{~L}(\alpha, \beta, \gamma)}{\partial \alpha^{2} \partial \beta} \\
& =\frac{-n}{\alpha^{2}}-\gamma \alpha^{\beta-2} \sum_{i=1}^{n} x_{i}^{-\beta}\left[(2 \beta-1)+\beta(\beta-1)\left(\log \alpha-\log \sum_{i=1}^{n} x_{i}\right)\right] \\
& =L_{121}=L_{211} \\
& L_{122}=\frac{\partial^{3} \mathrm{~L}(\alpha, \beta, \gamma)}{\partial \alpha \partial \beta^{2}} \\
& =-\gamma \alpha^{\beta-1} \sum_{i=1}^{n} x_{i}^{-\beta}\left(\log \alpha-\log \sum_{i=1}^{n} x_{i}\right)\left(2+\beta\left(\left(\log \alpha-\log \sum_{i=1}^{n} x_{i}\right)\right)^{2}\right) \\
& =L_{221}=L_{212} \\
& L_{123}=\frac{\partial^{3} \mathrm{~L}(\alpha, \beta, \gamma)}{\partial \alpha \partial \beta \partial \gamma} \\
& =-\alpha^{\beta-1} \sum_{i=1}^{n} x_{i}^{-\beta}\left(1+\beta\left(\log \alpha-\log \sum_{i=1}^{n} x_{i}\right)\right)=L_{321}=L_{213}
\end{aligned}
$$




\section{BAYESIAN ESTIMATION OF GIWD}

$$
\begin{gathered}
L_{133}=\frac{\partial^{3} \mathrm{~L}(\alpha, \beta, \gamma)}{\partial \alpha \partial \gamma^{2}}=0=L_{313}=L_{331} \\
L_{223}=\frac{\partial^{3} \mathrm{~L}(\alpha, \beta, \gamma)}{\partial \beta^{2} \partial \gamma} \\
=-\left(\log \alpha-\log \sum_{i=1}^{n} x_{i}\right)^{2} \alpha^{\beta} \sum_{i=1}^{n} x_{i}^{-\beta}=L_{322}=L_{323} \\
L_{233}=\frac{\partial^{3} \mathrm{~L}(\alpha, \beta, \gamma)}{\partial \beta \partial \gamma^{2}}=0=L_{332}=L_{323}
\end{gathered}
$$

The expression $a_{i}=\rho_{1} \sigma_{i 1}+\rho_{2} \sigma_{i 2}+\rho_{3} \sigma_{i 3}, i=1,2,3$, hence in this case

$$
\begin{aligned}
& a_{1}=\rho_{1} \sigma_{11}+\rho_{2} \sigma_{12}+\rho_{3} \sigma_{13} \\
& a_{2}=\rho_{1} \sigma_{21}+\rho_{2} \sigma_{22}+\rho_{3} \sigma_{23} \\
& a_{3}=\rho_{1} \sigma_{31}+\rho_{2} \sigma_{32}+\rho_{3} \sigma_{33}
\end{aligned}
$$

Using these general expressions of Lindley's approximation, Bayesian estimators for $\alpha, \beta, \gamma$ for different loss functions are derived below.

\section{Bayesian Estimators of $\alpha, \beta, y$ for Informative Prior using Lindley's Approximation in Case of Squared Error Loss Function (SELF)}

The squared error loss function is

$$
\mathrm{L}(\theta)=(\theta-\hat{\theta})^{2}
$$

where $\hat{\theta}$ is the estimator of $\theta$. The Bayes estimator of $\theta$ under the SELF is

$$
\hat{\theta}_{\mathrm{SELF}}=\mathrm{E}(\theta)
$$




\section{KAUR ET AL}

where expectation is taken with respect to posterior density. Note the Bayesian estimator of $\theta$ using Lindley's approximation is directly given by the expression $\mathrm{I}(x)$ in equation (12).

\section{Bayesian Estimator of a under Squared Error Loss Function}

In this case, $\mathrm{u}\left(\theta_{1}, \theta_{2}, \theta_{3}\right)=\mathrm{u}(\alpha, \beta, \gamma)$ is function of $\alpha$ only and it is given by $u_{\alpha \mathrm{s}}=\mathrm{u}(\alpha, \beta, \gamma)=\alpha$, since parameters $\alpha, \beta, \gamma$ are assumed to be independent. Hence, using equation (12),

$$
\mathrm{I}(x)=\mathrm{E}[\mathrm{u}(\alpha, \beta, \gamma)]=\mathrm{E}(\alpha)
$$

the first derivative of $u_{\alpha \mathrm{s}}$ with respect to $\alpha$ is

$$
u_{\alpha \mathrm{s} 1}=\frac{\partial u_{\alpha \mathrm{s}}}{\partial \alpha}=1
$$

all other $u_{i j}=0$ for $i, j=1,2,3$ because it is a function of $\alpha$ only. The expressions for the $a_{i}, i=1,2,3$ are given in equations (38)-(40), and

$$
\begin{aligned}
& a_{4}=\left(u_{12} \sigma_{12}+u_{13} \sigma_{13}+u_{23} \sigma_{23}\right)=0 \\
& a_{5}=\frac{1}{2}\left(u_{11} \sigma_{11}+u_{22} \sigma_{22}+u_{33} \sigma_{33}\right)=0
\end{aligned}
$$

Using equation (12), the expression for $\mathrm{I}(x)$ is derived as

$$
\mathrm{I}(x)=\hat{\alpha}+\left(u_{\alpha \mathrm{s} 1} a_{1}\right)+\frac{1}{2}\left[A \sigma_{11}+B \sigma_{21}+C \sigma_{31}\right]
$$

where

$$
\begin{aligned}
& A=\sigma_{11} L_{111}+2 \sigma_{12} L_{121}+2 \sigma_{13} L_{131}+2 \sigma_{23} L_{231}+\sigma_{22} L_{221}+\sigma_{33} L_{331} \\
& B=\sigma_{11} L_{112}+2 \sigma_{12} L_{122}+2 \sigma_{13} L_{132}+2 \sigma_{23} L_{232}+\sigma_{22} L_{222}+\sigma_{33} L_{332} \\
& C=\sigma_{11} L_{113}+2 \sigma_{12} L_{123}+2 \sigma_{13} L_{133}+2 \sigma_{23} L_{233}+\sigma_{22} L_{223}+\sigma_{33} L_{333}
\end{aligned}
$$




\section{BAYESIAN ESTIMATION OF GIWD}

The detailed expression for the various $L_{i j k}$ for $i, j, k=1,2,3$ are obtained in the above equations (28)-(37). Moreover, $\sigma_{i j}$ is the $(i, j)^{\text {th }}$ element of the inverse of the matrix of $L_{i j}$, i.e.

$$
\sigma_{i j}=\frac{-1}{L_{i j}}, \quad i, j=1,2
$$

where the $L_{i j}$ for $i, j=1,2$ are computed in equations (22)-(27) above. Therefore, the Bayesian estimator of $\alpha$ under the squared error loss function is

$$
\begin{aligned}
\hat{\alpha}_{\mathrm{SELF}}= & \hat{\alpha}+\left(\frac{a_{2}-1}{\hat{\alpha}}-b_{2}\right) \sigma_{11}+\left(\frac{a_{3}-1}{\hat{\beta}}-b_{3}\right) \sigma_{12}+\left(\frac{a_{1}-1}{\hat{\gamma}}-b_{1}\right) \sigma_{13} \\
& +\frac{1}{2}\left[A \sigma_{11}+B \sigma_{21}+C \sigma_{31}\right]
\end{aligned}
$$

In the similar way, Bayesian estimators of $\beta\left(\hat{\beta}_{\mathrm{SELF}}\right)$ and $\gamma\left(\hat{\gamma}_{\mathrm{SELF}}\right)$ under the squared error loss function are given as

$$
\begin{aligned}
\hat{\beta}_{\mathrm{SELF}}= & \hat{\beta}+\left(\frac{a_{2}-1}{\hat{\alpha}}-b_{2}\right) \sigma_{21}+\left(\frac{a_{3}-1}{\hat{\beta}}-b_{3}\right) \sigma_{22}+\left(\frac{a_{1}-1}{\hat{\gamma}}-b_{1}\right) \sigma_{23} \\
& +\frac{1}{2}\left[A \sigma_{12}+B \sigma_{22}+C \sigma_{32}\right] \\
\hat{\gamma}_{\mathrm{SELF}}= & \hat{\gamma}+\left(\frac{a_{2}-1}{\hat{\alpha}}-b_{2}\right) \sigma_{31}+\left(\frac{a_{3}-1}{\hat{\beta}}-b_{3}\right) \sigma_{32}+\left(\frac{a_{1}-1}{\hat{\gamma}}-b_{1}\right) \sigma_{33} \\
& +\frac{1}{2}\left[A \sigma_{13}+B \sigma_{23}+C \sigma_{33}\right]
\end{aligned}
$$

The squared error loss functions for $\alpha, \beta, \gamma$ are

$$
\begin{aligned}
& \mathrm{L}(\alpha)=\left(\alpha-\hat{\alpha}_{\mathrm{SELF}}\right)^{2} \\
& \mathrm{~L}(\beta)=\left(\beta-\hat{\beta}_{\mathrm{SELF}}\right)^{2} \\
& \mathrm{~L}(\gamma)=\left(\gamma-\hat{\gamma}_{\mathrm{SELF}}\right)^{2}
\end{aligned}
$$




\section{KAUR ET AL}

\section{Bayesian Estimators of $\beta$ and $y$ for Informative Prior using Lindley's Approximation in Case of Linear Exponential Loss Function (LINEX)}

The LINEX loss function is appropriate in case of shape parameter. So, it is not appropriate for $\alpha$, but for $\beta$ and $\gamma$ we make use of the LINEX loss function and obtain Bayesian estimators of $\beta$ and $\gamma$ under this set-up.

The LINEX loss function is given by

$$
\mathrm{L}(\hat{\theta}, \theta)=\mathrm{e}^{c(\hat{\theta}-\theta)}-c(\hat{\theta}-\theta)-1, \quad c \neq 0
$$

and the Bayes estimator of $\theta$ under the LINEX loss function is

$$
\hat{\theta}_{\mathrm{LINEX}}=-\frac{1}{c} \ln \left[\mathrm{E}\left(\mathrm{e}^{-c \theta}\right)\right], \quad c \neq 0
$$

Using Lindley's approximation, the Bayesian estimator of $\theta$ under the LINEX loss function is given by

$$
\hat{\theta}_{\mathrm{LINEX}}=-\frac{1}{c} \ln [\mathrm{I}(x)]
$$

where $\mathrm{I}(x)$ is the expression given by equation (12).

\section{Bayesian Estimator of $\beta$ under LINEX Loss Function}

The Bayesian estimator of $\beta$ under the LINEX loss function is given by

$$
\hat{\beta}_{\mathrm{LINEX}}=-\frac{1}{c} \ln \left[\mathrm{E}\left(\mathrm{e}^{-c \beta}\right)\right]
$$

Therefore

$$
\mathrm{I}(x)=\mathrm{E}[\mathrm{u}(\alpha, \beta, \gamma)]=\mathrm{E}\left(\mathrm{e}^{-c \beta}\right)
$$

In this case, $\mathrm{u}(\alpha, \beta, \gamma)$ is function of $\beta$ only and is defined as

$$
u_{\beta l}=\mathrm{u}(\alpha, \beta, \gamma)=\mathrm{e}^{-c \beta}
$$




\section{BAYESIAN ESTIMATION OF GIWD}

with the first and second derivatives of $u_{\beta l}$ with respect to $\beta$ given by

$$
\begin{aligned}
& u_{\beta l 2}=\frac{\partial u_{\beta l}}{\partial \beta}=-c \mathrm{e}^{-c \beta} \\
& u_{\beta l 22}=\frac{\partial^{2} u_{\beta l}}{\partial \beta^{2}}=c^{2} \mathrm{e}^{-c \beta}
\end{aligned}
$$

As $\mathrm{u}(\alpha, \beta, \gamma)$ is function of $\beta$,

$$
a_{4}=u_{12} \sigma_{12}+u_{13} \sigma_{13}+u_{23} \sigma_{23}=0
$$

and

$$
\begin{gathered}
a_{5}=\frac{1}{2}\left(u_{\beta l 22} \sigma_{22}\right)=\frac{c^{2} \mathrm{e}^{-c \beta}}{2} \sigma_{22} \\
\mathrm{I}(x)=\mathrm{u}(\hat{\alpha}, \hat{\beta}, \hat{\gamma})+u_{\beta l 2} a_{2}+a_{5}+\frac{u_{\beta l 2}}{2}\left[A \sigma_{12}+B \sigma_{22} C \sigma_{32}\right] \\
=\mathrm{e}^{-c \hat{\beta}}-c \mathrm{e}^{-c \hat{\beta}}\left(\left(\frac{a_{2}-1}{\hat{\alpha}}-b_{2}\right) \sigma_{21}+\left(\frac{a_{3}-1}{\hat{\beta}}-b_{3}\right) \sigma_{22}+\left(\frac{a_{3}-1}{\hat{\gamma}}-b_{1}\right) \sigma_{23}\right) \\
+\frac{c^{2} \mathrm{e}^{-c \hat{\beta}}}{2} \sigma_{22}-\frac{c \mathrm{e}^{-c \hat{\beta}}}{2}\left[A \sigma_{12}+B \sigma_{22} C \sigma_{32}\right] \\
=\mathrm{e}^{-c \hat{\beta}}\left\{1-c\left(\left(\frac{a_{2}-1}{\hat{\alpha}}-b_{2}\right) \sigma_{21}+\left(\frac{a_{3}-1}{\hat{\beta}}-b_{3}\right) \sigma_{22}+\left(\frac{a_{3}-1}{\hat{\gamma}}-b_{1}\right) \sigma_{23}\right)\right. \\
+ \\
\left.+\frac{c^{2}}{2} \sigma_{22}-\frac{c}{2}\left[A \sigma_{12}+B \sigma_{22} C \sigma_{32}\right]\right\}
\end{gathered}
$$

Hence, the Bayesian estimator of $\beta$ is given by

$$
\hat{\beta}_{\mathrm{LINEX}}=-\frac{1}{c} \ln \left[\mathrm{E}\left(\mathrm{e}^{-c \beta}\right)\right]
$$

Putting the value of $\mathrm{I}(x)$ in the above equation, 


$$
\begin{aligned}
\hat{\beta}_{\mathrm{LNEX}}= & \hat{\beta}-\frac{1}{c} \ln \left\{1-c\left[\left(\frac{a_{2}-1}{\hat{\alpha}}-b_{2}\right) \sigma_{21}+\left(\frac{a_{3}-1}{\hat{\beta}}-b_{3}\right) \sigma_{22}+\left(\frac{a_{3}-1}{\hat{\gamma}}-b_{1}\right) \sigma_{23}\right.\right. \\
& \left.\left.-\frac{c^{2}}{2} \sigma_{22}+\frac{c}{2}\left[A \sigma_{12}+B \sigma_{22} C \sigma_{32}\right]\right]\right\}
\end{aligned}
$$

The terms in the estimator are the same as explained earlier.

\section{Bayesian Estimator of Y under LINEX Loss Function}

$$
\begin{aligned}
\hat{\gamma}_{\mathrm{LINEX}}= & \hat{\gamma}-\frac{1}{c} \ln \left\{1-c\left[\left(\frac{a_{2}-1}{\hat{\alpha}}-b_{2}\right) \sigma_{31}+\left(\frac{a_{3}-1}{\hat{\beta}}-b_{3}\right) \sigma_{32}+\left(\frac{a_{3}-1}{\hat{\gamma}}-b_{3}\right) \sigma_{23}\right.\right. \\
& \left.\left.-\frac{c^{2}}{2} \sigma_{33}+\frac{c}{2}\left[A \sigma_{13}+B \sigma_{23} C \sigma_{33}\right]\right]\right\}
\end{aligned}
$$

The LINEX loss functions for $\beta$ and $\gamma$ are

$$
\begin{aligned}
\mathrm{L}\left(\hat{\beta}_{\mathrm{LINEX}}, \beta\right) & =\mathrm{e}^{c\left(\hat{\beta}_{\text {LINEX }}-\beta\right)}-c\left(\hat{\beta}_{\mathrm{LINEX}}-\beta\right)-1, \quad c \neq 0 \\
\mathrm{~L}\left(\hat{\gamma}_{\mathrm{LINEX}}, \gamma\right) & =\mathrm{e}^{c\left(\hat{\gamma}_{\text {INEX }}-\gamma\right)}-c\left(\hat{\gamma}_{\mathrm{LINEX}}-\gamma\right)-1, \quad c \neq 0
\end{aligned}
$$

\section{Bayesian Estimators of $\alpha$ for Informative Prior using Lindley's Approximation in Case of General Entropy Loss Function (GELF)}

The LINEX loss function is not as appropriate for estimation of scale parameter as it is for shape parameter (Basu \& Ebrahimi, 1991; Parsian, Sanjari, \& Nematollahi, 1993). So, for estimation of $\alpha$, an entropy loss function is used.

The general entropy loss function is defined as

$$
\mathrm{L}(\theta, \hat{\theta}) \propto\left(\frac{\hat{\theta}}{\theta}\right)^{b_{\mathrm{c}}}-b_{\mathrm{e}} \ln \left(\frac{\hat{\theta}}{\theta}\right)-1
$$

The Bayes estimator of $\theta$ under general entropy loss is given as 


\section{BAYESIAN ESTIMATION OF GIWD}

$$
\hat{\theta}_{\mathrm{GELF}}=\left[\mathrm{E}_{\theta}\left(\theta^{-b_{\mathrm{e}}}\right)\right]^{\frac{-1}{b_{\mathrm{e}}}}
$$

Using Lindley's approximation method, the Bayesian estimator of $\theta$ under the general entropy loss function is

$$
\hat{\theta}_{\mathrm{GELF}}=[\mathrm{I}(x)]^{\frac{-1}{b_{\mathrm{e}}}}
$$

\section{Bayesian Estimator of a under General Entropy Loss Function}

The Bayesian estimator of $\alpha$ under the general entropy loss function is given by

$$
\begin{gathered}
\hat{\alpha}_{\mathrm{GELF}}=\left[\mathrm{E}\left(\alpha^{-b_{\mathrm{e}}}\right)\right]^{\frac{-1}{b_{\mathrm{e}}}} \\
\mathrm{I}(x)=\mathrm{E}[\mathrm{u}(\alpha, \beta, \gamma)]=\mathrm{E}\left(\alpha^{-b_{\mathrm{e}}}\right)
\end{gathered}
$$

is function of $\alpha$ only, and $\mathrm{u}(\alpha, \beta, \gamma)$ is denoted by $u_{\alpha \mathrm{e}}$, i.e.

$$
u_{\alpha \mathrm{e}}=\mathrm{u}(\alpha, \beta, \gamma)=\alpha^{-b_{\mathrm{e}}}
$$

The first and second derivatives of $u_{\alpha \mathrm{e}}$ with respect to $\alpha$ are given by

$$
\begin{gathered}
u_{\alpha \mathrm{e} 1}=\frac{\partial u_{\alpha l}}{\partial \alpha}=-b_{\mathrm{e}} \alpha^{-b_{\mathrm{e}}-1} \\
u_{\alpha \mathrm{e} 11}=\frac{\partial^{2} u_{\alpha l}}{\partial \alpha^{2}}=b_{\mathrm{e}}\left(b_{\mathrm{e}}+1\right) \alpha^{-b_{\mathrm{e}}-2} \\
a_{5}=\frac{1}{2}\left(u_{\alpha \mathrm{e} 11} \sigma_{11}\right)=\frac{b_{\mathrm{e}}\left(b_{\mathrm{e}}+1\right) \alpha^{-b_{\mathrm{e}}-2}}{2} \sigma_{11} \\
\mathrm{I}(x)=\mathrm{E}\left(\alpha^{-b_{\mathrm{e}}}\right) \\
=\mathrm{u}(\hat{\alpha}, \hat{\beta}, \hat{\gamma})+u_{\alpha \mathrm{e} 1} a_{1}+a_{5}+\frac{1}{2}\left[A u_{\alpha \mathrm{e} 1} \sigma_{11}+B u_{\alpha \mathrm{e} 1} \sigma_{21}+C u_{\alpha \mathrm{e} 1} \sigma_{31}\right]
\end{gathered}
$$




$$
\begin{aligned}
= & \hat{\alpha}^{-b_{\mathrm{e}}}-b_{\mathrm{e}} \hat{\alpha}^{-b_{\mathrm{e}}-1}\left(\left(\frac{a_{2}-1}{\hat{\alpha}}-b_{2}\right) \sigma_{11}+\left(\frac{a_{3}-1}{\hat{\beta}}-b_{3}\right) \sigma_{12}+\left(\frac{a_{1}-1}{\hat{\gamma}}-b_{1}\right) \sigma_{13}\right) \\
& +\frac{b_{\mathrm{e}}\left(b_{\mathrm{e}}+1\right) \hat{\alpha}^{-b_{\mathrm{e}}-2}}{2} \sigma_{11}-\frac{b_{\mathrm{e}} \hat{\alpha}^{-b_{\mathrm{e}}-1}}{2}\left[A \sigma_{11}+B \sigma_{21}+C \sigma_{31}\right] \\
= & \left(\hat { \alpha } ^ { - b _ { \mathrm { e } } } \left\{1-\frac{b_{\mathrm{e}}}{\hat{\alpha}}\left[\left(\left(\frac{a_{2}-1}{\hat{\alpha}}-b_{2}\right) \sigma_{11}+\left(\frac{a_{3}-1}{\hat{\beta}}-b_{3}\right) \sigma_{12}+\left(\frac{a_{1}-1}{\hat{\gamma}}-b_{1}\right) \sigma_{13}\right.\right.\right.\right. \\
& \left.\left.\left.\left.-\frac{\left(b_{\mathrm{e}}+1\right) \sigma_{11}}{2 \hat{\alpha}}\right]+\frac{1}{2}\left[A \sigma_{11}+B \sigma_{21}+C \sigma_{31}\right]\right)\right\}\right)
\end{aligned}
$$

The terms in $\mathrm{I}(x)$ are same as explained earlier. Therefore, the Bayesian estimator of $\alpha$ under the general entropy loss function is given by

$$
\begin{aligned}
\hat{\alpha}_{\mathrm{GELF}}= & {[\mathrm{I}(x)]^{\frac{-1}{b_{\mathrm{e}}}} } \\
= & \left(\hat { \alpha } ^ { - b _ { \mathrm { e } } } \left\{1-\frac{b_{\mathrm{e}}}{\hat{\alpha}}\left[\left(\frac{a_{2}-1}{\hat{\alpha}}-b_{2}\right) \sigma_{11}+\left(\frac{a_{3}-1}{\hat{\beta}}-b_{3}\right) \sigma_{12}+\left(\frac{a_{1}-1}{\hat{\gamma}}-b_{1}\right) \sigma_{13}\right.\right.\right. \\
& \left.\left.\left.-\frac{\left(b_{\mathrm{e}}+1\right) \sigma_{11}}{2 \hat{\alpha}}\right]+\frac{1}{2}\left[A \sigma_{11}+B \sigma_{21}+C \sigma_{31}\right]\right\}\right)^{\frac{-1}{b_{\mathrm{e}}}}
\end{aligned}
$$

The general entropy loss function for $\alpha$ is

$$
\mathrm{L}\left(\alpha, \hat{\alpha}_{\mathrm{GELF}}\right) \propto\left(\frac{\hat{\alpha}_{\mathrm{GELF}}}{\alpha}\right)^{b_{\mathrm{e}}}-b_{\mathrm{e}} \ln \left(\frac{\hat{\alpha}_{\mathrm{GELF}}}{\alpha}\right)-1
$$

\section{Bayesian Estimators of $\alpha, \beta$, and $y$ using Non-Informative Prior (Jeffreys' Prior)}

Referring to equation (11), the joint posterior distribution for $\alpha, \beta, \gamma$ using Jeffreys' prior, i.e. non-informative prior, is given by 


\section{BAYESIAN ESTIMATION OF GIWD}

$$
\mathrm{g}_{\mathrm{J}}^{*}(\alpha, \beta, \gamma \mid x)=\frac{\gamma^{n-1} \beta^{n-1} \alpha^{n \beta-1} \prod_{i=1}^{n} x_{i}^{-(\beta+1)} \exp \left(-\gamma \alpha^{\beta} \sum_{i=1}^{n} x_{i}^{-\beta}\right)}{\iiint_{0}^{\infty} \gamma^{n-1} \beta^{n-1} \alpha^{n \beta-1} \prod_{i=1}^{n} x_{i}^{-(\beta+1)} \exp \left(-\gamma \alpha^{\beta} \sum_{i=1}^{n} x_{i}^{-\beta}\right) \partial \alpha \partial \beta \partial \gamma}
$$

The form of posterior distribution is complex, so the evaluation of the Bayesian estimators of $\alpha, \beta, \gamma$ is tedious. Therefore, using Lindley's approximation method, the Bayesian estimators of the parameters are obtained in the case of symmetrical and asymmetrical loss functions for Jeffreys' prior. The expression for Bayesian estimators in this case will also depend upon the various loss functions.

(i) Using Lindley's approximation, Bayesian estimators of $\alpha, \beta, \gamma$ under squared error loss function is

$$
\begin{aligned}
& \hat{\alpha}_{\text {SELF }}=\mathrm{I}(x)=\mathrm{E}[\mathrm{u}(\alpha, \beta, \gamma)]=\mathrm{E}(\alpha) ; \mathrm{u}(\alpha, \beta, \gamma) \text { is a function of } \alpha \\
& \hat{\beta}_{\text {SELF }}=\mathrm{I}(x)=\mathrm{E}[\mathrm{u}(\alpha, \beta, \gamma)]=\mathrm{E}(\beta) ; \mathrm{u}(\alpha, \beta, \gamma) \text { is a function of } \beta \\
& \hat{\gamma}_{\mathrm{SELF}}=\mathrm{I}(x)=\mathrm{E}[\mathrm{u}(\alpha, \beta, \gamma)]=\mathrm{E}(\gamma) ; \mathrm{u}(\alpha, \beta, \gamma) \text { is a function of } \gamma
\end{aligned}
$$

where

$$
\begin{aligned}
\mathrm{I}(x)= & \mathrm{E}[\mathrm{u}(\alpha, \beta, \gamma)] \\
= & \mathrm{u}(\hat{\alpha}, \hat{\beta}, \hat{\gamma})+\left(u_{1} a_{1}+u_{2} a_{2}+u_{3} a_{3}+a_{4}+a_{5}\right)+\frac{1}{2}\left[A\left(u_{1} \sigma_{11}+u_{2} \sigma_{12}+u_{3} \sigma_{13}\right)\right. \\
& \left.+B\left(u_{1} \sigma_{21}+u_{2} \sigma_{22}+u_{3} \sigma_{23}\right)+C\left(u_{1} \sigma_{31}+u_{2} \sigma_{32}+u_{3} \sigma_{33}\right)\right]
\end{aligned}
$$

The expression of $\rho$ depends on prior density, therefore $\rho$ is computed for noninformative prior and given below.

The log of joint prior density $(\rho)$ in the case of non-informative prior, referring to equation (10), is

$$
\begin{aligned}
& \rho=\log \left(\mathrm{g}_{\mathrm{J}}(\alpha, \beta, \gamma)\right)=-\log \gamma-\log \alpha-\log \beta \\
& \rho_{1}=\frac{\partial \rho}{\partial \alpha}=-\frac{1}{\alpha} \\
& \rho_{2}=\frac{\partial \rho}{\partial \beta}=-\frac{1}{\beta}
\end{aligned}
$$




\section{KAUR ET AL}

$$
\rho_{3}=\frac{\partial \rho}{\partial \gamma}=-\frac{1}{\gamma}
$$

(ii) The Bayesian estimators of $\beta$ and $\gamma$ under the LINEX loss function are given by

$$
\begin{aligned}
& \hat{\beta}_{\text {LINEX }}=-\frac{1}{c} \ln [\mathrm{I}(x)], \mathrm{I}(x)=\mathrm{E}\left(e^{-c \beta}\right) \\
& \hat{\gamma}_{\text {LINEX }}=-\frac{1}{c} \ln [\mathrm{I}(x)], \mathrm{I}(x)=\mathrm{E}\left(e^{-c \gamma}\right)
\end{aligned}
$$

(iii) The Bayesian estimator of $\alpha$ under the general entropy loss function is given by

$$
\hat{\alpha}_{\mathrm{GELF}}=[\mathrm{I}(x)]^{\frac{-1}{b_{\mathrm{e}}}}, \mathrm{I}(x)=\mathrm{E}\left(\alpha^{-b_{\mathrm{e}}}\right)
$$

The terms in $\mathrm{I}(x)$ are the same as already explained.

Table 1. Bayesian estimators of $\alpha, \beta, \gamma$ using Jeffreys' prior

Param.

Estimators of $\alpha, \beta, \gamma$ under SELF, LINEX and GELF

$$
\hat{\alpha}_{\mathrm{SELF}}=\hat{\alpha}-\frac{\sigma_{11}}{\hat{\alpha}}-\frac{\sigma_{12}}{\hat{\beta}}-\frac{\sigma_{13}}{\hat{\gamma}}+\frac{1}{2}\left[A \sigma_{11}+B \sigma_{21}+C \sigma_{31}\right]
$$

$\alpha$

$$
\begin{array}{r}
\hat{\alpha}_{\mathrm{GELF}}=\left(\hat{\alpha}^{-b_{\circ}}\left\{1+\frac{b_{\mathrm{e}}}{\hat{\alpha}}\left[\frac{\sigma_{11}}{\hat{\alpha}}+\frac{\sigma_{12}}{\hat{\beta}}+\frac{\sigma_{13}}{\hat{\gamma}}+\frac{\left(b_{\mathrm{e}}+1\right) \sigma_{33}}{2 \hat{\alpha}}\right]+\frac{u_{\mathrm{ae} 1}}{2}\left[A \sigma_{11}+B \sigma_{21}+C \sigma_{31}\right]\right\}\right)^{\frac{-1}{b_{0}}} \\
\hat{\beta}_{\mathrm{SELF}}=\hat{\beta}-\frac{\sigma_{21}}{\hat{\alpha}}-\frac{\sigma_{22}}{\hat{\beta}}-\frac{\sigma_{23}}{\hat{\gamma}}+\frac{1}{2}\left[A \sigma_{12}+B \sigma_{22}+C \sigma_{32}\right]
\end{array}
$$

$\beta$

$$
\begin{array}{r}
\hat{\beta}_{\text {LINEX }}=\left(\hat{\beta}-\frac{1}{c} \ln \left\{1+c\left[\frac{\sigma_{21}}{\hat{\alpha}}+\frac{\sigma_{22}}{\hat{\beta}}+\frac{\sigma_{23}}{\hat{\gamma}}+\frac{c}{2} \sigma_{22}-\frac{1}{2}\left[A \sigma_{12}+B \sigma_{22}+C \sigma_{32}\right]\right]\right\}\right) \\
\hat{V}_{\text {SELF }}=\hat{\gamma}-\frac{\sigma_{31}}{\hat{\alpha}}-\frac{\sigma_{32}}{\hat{\beta}}-\frac{\sigma_{33}}{\hat{\gamma}}+\frac{1}{2}\left[A \sigma_{13}+B \sigma_{23}+C \sigma_{33}\right] \\
\hat{\mathrm{LINEX}}=\left(\hat{\gamma}-\frac{1}{c} \ln \left\{1+c\left[\frac{\sigma_{31}}{\hat{\alpha}}+\frac{\sigma_{32}}{\hat{\beta}}+\frac{\sigma_{33}}{\hat{\gamma}}+\frac{c}{2} \sigma_{33}-\frac{1}{2}\left[A \sigma_{13}+B \sigma_{23}+C \sigma_{33}\right]\right]\right\}\right)
\end{array}
$$




\section{BAYESIAN ESTIMATION OF GIWD}

By using these expressions of different loss functions, i.e. the squared error loss function, LINEX loss function, and general entropy loss function, Bayesian estimators of $\alpha, \beta, \gamma$ are obtained for non-informative prior and presented in Table 1.

\section{Semi-Bayesian Approach}

Generalized maximum likelihood estimators are obtained for the parameters of GIWD using informative and non-informative prior. It is known generalized maximum likelihood estimators are maximum likelihood estimators of the posterior distribution.

Generalized Maximum Likelihood Estimators of Parameters $(\alpha, \beta, y)$ of Generalized Inverse Weibull Distribution using Informative Prior

Assuming independence among parameters, the posterior distribution using informative prior is given by

$$
\begin{aligned}
& \mathrm{g}_{\mathrm{I}}^{*}(\alpha, \beta, \gamma \mid x) \\
& \quad \propto \gamma^{n+a_{1}-1} \beta^{n+a_{3}-1} \alpha^{n \beta+a_{2}-1} \prod_{i=1}^{n} x_{i}^{-(\beta+1)} \exp \left(\left(-\gamma \alpha^{\beta} \sum_{i=1}^{n} x_{i}^{-\beta}\right)-\gamma b_{1}-\alpha b_{2}-\beta b_{3}\right)
\end{aligned}
$$

The log of the posterior distribution is

$$
\begin{aligned}
\ln \left(\mathrm{g}_{\mathrm{I}}^{*}(\alpha, \beta, \gamma \mid x)\right)= & \left(n+a_{1}-1\right) \ln (\gamma)+\left(n+a_{3}-1\right) \ln (\beta)+\left(n \beta+a_{2}-1\right) \ln (\alpha) \\
& -(\beta+1) \prod_{i=1}^{n} \ln \left(x_{i}\right)-\gamma \alpha^{\beta} \sum_{i=1}^{n} x_{i}^{-\beta}-b_{1} \gamma-b_{3} \beta-b_{2} \alpha
\end{aligned}
$$

Taking partial derivatives with respect to $\alpha, \beta$, and $\gamma$ gives likelihood equations

$$
\frac{\partial \ln \left(\mathrm{g}_{\mathrm{I}}^{*}(\alpha, \beta, \gamma \mid x)\right)}{\partial a}=\frac{n \beta+a_{2}-1}{\alpha}-\beta \sum_{i=1}^{n} x_{i}^{-\beta}-b_{2}=0
$$




\section{KAUR ET AL}

$$
\begin{aligned}
& \frac{\partial \ln \left(\mathrm{g}_{\mathrm{I}}^{*}(\alpha, \beta, \gamma \mid x)\right)}{\partial \beta} \\
& =\frac{n+a_{3}-1}{\beta}+n \ln (\gamma)-\ln \left(\sum_{i=1}^{n} x_{i}\right)-\gamma \alpha^{\beta} \sum_{i=1}^{n} x_{i}^{-\beta}\left(\ln \gamma-\ln \left(\sum_{i=1}^{n} x_{i}\right)\right)-b_{3} \\
& =0 \\
& \qquad \frac{\partial \ln \left(\mathrm{g}_{\mathrm{I}}^{*}(\alpha, \beta, \gamma \mid x)\right)}{\partial \gamma}=\frac{n+a_{1}-1}{\gamma}-\alpha^{\beta} \sum_{i=1}^{n} x_{i}^{-\beta}-b_{1}=0
\end{aligned}
$$

By solving these three equations simultaneously, the GMLE of $\alpha, \beta$, and $\gamma$ are obtained. As the expressions are not in explicit form, one may use various numerical methods to obtain the estimators, e.g. Newton Raphson's method (Raphson, 1690) or the Optim function in R (Nash, 1990). In the present study, the latter approach, i.e. Optim function in $\mathrm{R}$, is used to obtain the estimators of the parameters $(\alpha, \beta, \gamma)$ of GIWD.

Note: Generalized maximum likelihood estimators of parameters $(\alpha, \beta, \gamma)$ of the GIWD using Jeffreys' prior are computed in a similar manner by taking the values of the hyperparameters to be zero $\left(a_{1}=b_{1}=a_{2}=b_{2}=a_{3}=b_{3}=0\right)$.

\section{Optimization of Hyperparameters}

The hyperparameters, the parameters used in the informative prior, also play an important role in the simulation exercise. So, the choice of hyperparameters is crucial and important to any simulation study. In literature, there are many approaches to estimate these hyperparameters, like using their maximum likelihood estimators, choosing hyperparameters randomly, min/max approach, estimating from past data, or the method of elicitation using a prior predicative distribution (Sinha \& Howlander, 1980; Ali, Aslam, Abbas, \& Kazmi, 2012; Aslam, 2003). In the previous sections, the min/max approach (Sinha \& Howlander, 1980) is used to check the robustness of hyperparameters. In the present section, a scaled total misfit technique is used, which leads to the best fitted hyperparameters out of random choice of some combination of hyperparameters with the parameters of distribution.

\section{A Scaled Total Misfit Measure (Park, 2005)}

First define a scaled misfit measure from a quantile estimation method as follows: 


\section{BAYESIAN ESTIMATION OF GIWD}

$$
A=\sum_{q} \frac{\left(\hat{x}_{q}-x_{q}\right)^{2}}{x_{q}}
$$

where $x_{q}$ is the true value for the $q$-quantile and $\hat{x}_{q}$ is the estimated $q$-quantile computed from GIWD. This measure can be viewed as the chi-square goodness-offit criterion for numeric estimates of quantiles.

Now the above misfit measure is extended to various sample sizes. This measure is computed for $N$, the number of Monte Carlo samples for different sample sizes $n$ (denoted by $N_{n}$ ), and then averaged:

$$
B_{n}=\sum_{i=1}^{N_{n}} \frac{A_{i}}{N_{n}}
$$

Here, $A_{i}$ is measure computed from the $i^{\text {th }}$ Monte Carlo sample.

Define, for a distribution, scaled total misfit measure (STMM) as a function of a given prior and estimation method:

$$
\mathrm{Q}(\pi, \mathrm{M})=\sum_{\theta} \mathrm{Q}_{\theta}(\pi, \mathrm{M})
$$

where $\pi$ is the prior, $\mathrm{M}$ is the method of estimation of the parameter $\theta$, and

$$
\begin{aligned}
\mathrm{Q}_{\theta}(\pi, \mathrm{M}) & =\sum_{n} B_{n} \\
& =\frac{\sum_{n} \sum_{i=1}^{N_{n}} \sum_{q} \frac{\left(\hat{x}_{q}-x_{q}\right)^{2}}{x_{q}}}{N_{n}}
\end{aligned}
$$

$\theta$ is the parameters of the distribution we are using for quantile estimation. Choose the best prior $(\pi)$ and $\mathrm{M}$ which minimizes $\mathrm{Q}(\pi, \mathrm{M})$ among serval M's and classes of priors $(\pi)$.

\section{Simulation Study}

A simulation study is conducted in two parts: firstly, for the selection of the best hyperparameters as per scaled total misfit measure; and secondly to see the relative 


\section{KAUR ET AL}

efficiency of Bayesian, semi-Bayesian (GMLE's), and non-Bayesian (MLE's) approach. The coding and the analysis are performed using the $\mathrm{R}$ programming language.

Random variables from GIWD have been generated using the transformation

$$
t=\alpha\left[\frac{-\ln (u)}{\gamma}\right]^{\frac{-1}{\beta}}
$$

where $u$ has uniform $\mathrm{U}(0,1)$ distribution.

Table 2. Misfit measure for $\alpha$

\begin{tabular}{rrrrrrrrrr}
$\boldsymbol{a}_{\mathbf{2}}=\boldsymbol{b}_{\mathbf{2}}$ & $\boldsymbol{\alpha} \mathbf{\boldsymbol { r }} \mathbf{0 . 5}$ & $\mathbf{1 . 0}$ & $\mathbf{1 . 5}$ & $\mathbf{2 . 0}$ & $\mathbf{2 . 5}$ & $\mathbf{3 . 0}$ & $\mathbf{3 . 5}$ & $\mathbf{4 . 0}$ & sum $\mathbf{Q}(\boldsymbol{\pi}, \mathbf{M})$ \\
\hline 1 & 10.48 & 12.76 & 8.48 & 7.67 & 8.66 & 8.43 & 7.80 & 9.55 & 73.83 \\
2 & 9.49 & 9.36 & 8.16 & 7.82 & 7.92 & 9.14 & 9.08 & 10.24 & 71.21 \\
3 & 12.00 & 9.65 & 10.61 & 6.95 & 7.18 & 8.70 & 10.33 & 9.81 & 75.23 \\
4 & 9.78 & 8.65 & 7.38 & 8.84 & 7.04 & 10.13 & 8.17 & 7.38 & 67.37 \\
6 & 9.29 & 8.82 & 8.96 & 9.44 & 10.59 & 6.45 & 7.22 & 6.82 & 67.59 \\
8 & 8.99 & 8.49 & 7.91 & 12.01 & 8.12 & 9.23 & 8.13 & 8.26 & 71.14 \\
\hline
\end{tabular}

Table 3. Misfit measure for $\beta$

\begin{tabular}{rrrrrrrrrr}
$\boldsymbol{a}_{\mathbf{3}}=\boldsymbol{b}_{\mathbf{3}}$ & $\boldsymbol{\beta} \mathbf{0 . 5}$ & $\mathbf{1 . 0}$ & $\mathbf{1 . 5}$ & $\mathbf{2 . 0}$ & $\mathbf{2 . 5}$ & $\mathbf{3 . 0}$ & $\mathbf{3 . 5}$ & $\mathbf{4 . 0}$ & sum $\mathbf{Q}(\boldsymbol{\pi}, \mathbf{M})$ \\
\hline 1 & 64.18 & 26.44 & 3.99 & 2.09 & 2.27 & 1.21 & 0.89 & 0.72 & 101.79 \\
2 & 56.74 & 21.33 & 6.60 & 3.16 & 2.60 & 1.44 & 1.06 & 0.82 & 93.75 \\
3 & 31.36 & 38.56 & 7.49 & 3.65 & 2.17 & 1.90 & 1.26 & 1.06 & 87.45 \\
4 & 40.40 & 24.98 & 6.48 & 2.93 & 4.44 & 1.92 & 1.37 & 0.86 & 83.38 \\
6 & 41.20 & 37.52 & 8.72 & 2.57 & 1.61 & 2.05 & 1.89 & 1.87 & 97.43 \\
8 & 33.47 & 42.70 & 9.08 & 6.09 & 3.72 & 3.49 & 2.01 & 1.53 & 102.09 \\
\hline
\end{tabular}

Table 4. Misfit measure for $y$

\begin{tabular}{rrrrrrrrrr}
$\boldsymbol{a}_{\mathbf{1}}=\boldsymbol{b}_{\mathbf{1}}$ & $\boldsymbol{\gamma} \mathbf{r} \mathbf{0 . 5}$ & $\mathbf{1 . 0}$ & $\mathbf{1 . 5}$ & $\mathbf{2 . 0}$ & $\mathbf{2 . 5}$ & $\mathbf{3 . 0}$ & $\mathbf{3 . 5}$ & $\mathbf{4 . 0}$ & sum $\mathbf{Q}(\boldsymbol{\pi}, \mathbf{M})$ \\
\hline 1 & 6.99 & 7.39 & 10.36 & 8.43 & 7.59 & 6.92 & 7.46 & 8.64 & 63.78 \\
2 & 7.31 & 8.57 & 7.39 & 7.18 & 8.70 & 7.28 & 6.17 & 6.57 & 59.17 \\
3 & 8.91 & 8.01 & 8.30 & 8.35 & 8.42 & 6.79 & 9.04 & 7.83 & 65.65 \\
4 & 8.93 & 10.38 & 8.71 & 8.38 & 7.88 & 10.95 & 6.47 & 7.54 & 69.24 \\
6 & 8.03 & 7.76 & 8.17 & 8.76 & 6.02 & 7.73 & 5.76 & 6.58 & 58.81 \\
8 & 10.61 & 9.77 & 7.61 & 8.13 & 8.89 & 8.03 & 7.91 & 6.30 & 67.25 \\
\hline
\end{tabular}




\section{BAYESIAN ESTIMATION OF GIWD}

The different sample sizes $n=10,20,40,80,100,150$ and quantiles $q=0.1$, $0.2, \ldots, 0.9$ are used to compute misfit measure, and $N_{n}=1000$ for different combinations of parameters and hyperparameters. The method used in misfit measure is the generalized maximum likelihood estimator and tables for the three parameters are given in Tables 2-4.

From Tables 2-4, the hyperparameter having minimum sum are selected for the further study:

(i) optimum selection of hyperparameters for $\alpha$ is $a_{2}=b_{2}=4$ (from Table 2)

(ii) optimum selection of hyperparameters for $\beta$ is $a_{3}=b_{3}=4$ (from Table 3)

(iii) optimum selection of hyperparameters for $\gamma$ is $a_{1}=b_{1}=6$ (from Table 4)

\section{Relative Efficiency of Various Approaches}

The mean square errors are computed using maximum likelihood, generalized maximum likelihood, and Bayesian with Jeffreys' and informative prior for the parameters which are obtained using squared error loss function, LINEX loss function, and general entropy loss function. These estimated losses are computed using 10,000 Monte Carlo simulations for different sample sizes $n=30,50,100$ with parameter combinations $\alpha=\beta=\gamma=2.5,4$. The combinations of hyperparameters are

(i) $a_{2}=b_{2}=4$ for $\alpha$,

(ii) $a_{3}=b_{3}=4$ for $\beta$,

(iii) $a_{1}=b_{1}=6$ for $\gamma$

taken for the simulation study according to misfit measure.

\section{Comparison of Non-Bayesian Approach (MLE's) and Semi-Bayesian Approach (GMLE's)}

The mean square errors of estimators using non-Bayesian approach (MLE's) and semi-Bayesian approach (GMLE's) are computed for parameters of GIWD and presented in Tables 5-7. 


\section{KAUR ET AL}

Table 5. Mean square error for $\alpha(\beta=\gamma=2.5)$

\begin{tabular}{rrrrr} 
& & & \multicolumn{2}{c}{ GMLE } \\
\cline { 4 - 5 } $\boldsymbol{n}$ & $\boldsymbol{\alpha}$ & MLE & Jeffreys' prior & Informative prior $^{\star}$ \\
\hline 30 & 2.5 & 0.03709354 & 0.03585720 & 0.03593395 \\
& 4.0 & 0.10401380 & 0.09482988 & 0.09537666 \\
50 & 2.5 & 0.02950446 & 0.02102547 & 0.02127104 \\
& 4.0 & 0.05882332 & 0.05315694 & 0.05396281 \\
100 & 2.5 & 0.01965800 & 0.01012477 & 0.01024923 \\
& 4.0 & 0.02736852 & 0.02606220 & 0.02612554 \\
\hline
\end{tabular}

Note: ${ }^{*} a_{2}=b_{2}=4$

Table 6. Mean square error for $\beta(\alpha=\gamma=2.5)$

GMLE

\begin{tabular}{rrrrr}
$\boldsymbol{n}$ & $\boldsymbol{\alpha}$ & MLE & Jeffreys' prior & Informative prior $^{\star}$ \\
\hline 30 & 2.5 & 0.93413690 & 0.66481748 & 0.72403600 \\
& 4.0 & 3.26021101 & 1.71687667 & 1.88861660 \\
50 & 2.5 & 0.83981996 & 0.64929604 & 0.71147050 \\
& 4.0 & 2.70095544 & 1.68945720 & 1.82528550 \\
100 & 2.5 & 0.76733404 & 0.63749605 & 0.69391660 \\
& 4.0 & 2.25106582 & 1.61900318 & 1.78274770 \\
\hline
\end{tabular}

Note: ${ }^{*} a_{3}=b_{3}=4$

Table 7. Mean square error for $\gamma(\alpha=\beta=2.5)$

\begin{tabular}{rrrrr} 
& & & \multicolumn{2}{c}{ GMLE } \\
\cline { 4 - 5 } $\boldsymbol{n}$ & $\boldsymbol{\alpha}$ & MLE & Jeffreys' prior & Informative prior $^{\boldsymbol{*}}$ \\
\hline 30 & 2.5 & 0.33456618 & 0.21560620 & 0.24933030 \\
& 4.0 & 0.98070166 & 0.55235300 & 0.63657320 \\
50 & 2.5 & 0.18460151 & 0.12808220 & 0.14161840 \\
& 4.0 & 0.90368559 & 0.32289400 & 0.35839910 \\
100 & 2.5 & 0.07845281 & 0.06577740 & 0.06599840 \\
& 4.0 & 0.32742309 & 0.26368640 & 0.31544770 \\
\hline
\end{tabular}

Note: * $a_{1}=b_{1}=6$

From Tables 5-7, it is observed that

(i) GMLE's using Jeffreys' prior and informative prior have less mean square error in comparison with MLEs, i.e. semi-Bayesian approaches (GMLEs) give better estimators than non-Bayesian approaches (MLEs). 


\section{BAYESIAN ESTIMATION OF GIWD}

(ii) In the case of the semi-Bayesian approach, GMLEs using Jeffreys' prior perform better in comparison with informative prior as they have smaller mean square error for all the parameters.

Table 8. Mean square error for $\alpha(\beta=\gamma=2.5)$

\begin{tabular}{rrrrrr} 
& & & \multicolumn{3}{c}{ Informative prior } \\
\cline { 4 - 6 } $\boldsymbol{n}$ & & $\boldsymbol{\alpha}$ & Jeffreys' prior & $\boldsymbol{a}_{\mathbf{3}}=\boldsymbol{b}_{\mathbf{3}}=\mathbf{4}$ & $\boldsymbol{c}=\mathbf{1}$ \\
\cline { 4 - 6 } 30 & 2.5 & 0.00075143 & 0.00018920 & 0.00004106 & 0.00012588 \\
& 4.0 & 0.03746346 & 0.01278650 & 0.00486047 & 0.00194826 \\
50 & 2.5 & 0.00045097 & 0.00012100 & $2.398339 \mathrm{E}-05$ & $7.276694 \mathrm{E}-05$ \\
& 4.0 & 0.00978687 & 0.00500640 & 0.00314538 & 0.00053285 \\
100 & 2.5 & $7.648816 \mathrm{E}-05$ & 0.00004520 & $4.668325 \mathrm{E}-06$ & $2.468399 \mathrm{E}-05$ \\
& 4.0 & 0.00385535 & 0.00075750 & $9.676239 \mathrm{E}-05$ & $5.809497 \mathrm{E}-05$ \\
\hline
\end{tabular}

Table 9. Mean square error for $\beta(\alpha=\gamma=2.5)$

\begin{tabular}{|c|c|c|c|c|c|}
\hline \multirow[b]{3}{*}{$n$} & \multirow[b]{3}{*}{$\alpha$} & \multirow[b]{3}{*}{ Jeffreys' prior } & \multicolumn{3}{|c|}{ Informative prior } \\
\hline & & & \multirow{2}{*}{$\begin{array}{r}\text { SELF } \\
a_{3}=b_{3}=4\end{array}$} & \multicolumn{2}{|c|}{ LINEX } \\
\hline & & & & $c=1$ & $c=-1$ \\
\hline \multirow[t]{2}{*}{30} & 2.5 & 0.01394930 & 0.00216250 & 0.00145047 & 0.00125890 \\
\hline & 4.0 & 1.61635760 & 1.59734500 & 0.15938417 & 0.35485000 \\
\hline \multirow[t]{2}{*}{50} & 2.5 & 0.01186860 & 0.00061610 & 1.653527E-04 & $1.352328 \mathrm{E}-04$ \\
\hline & 4.0 & 1.60876570 & 1.50787870 & 0.00193841 & 0.00128650 \\
\hline \multirow[t]{2}{*}{100} & 2.5 & 4.107887E-03 & 0.00016530 & 1.119729E-04 & 1.092251E-04 \\
\hline & 4.0 & 1.57676760 & 1.46878800 & 0.00013869 & 0.00013300 \\
\hline
\end{tabular}

Table 10. Mean square error for $\gamma(\alpha=\beta=2.5)$

\begin{tabular}{rrrrrr} 
& & & \multicolumn{3}{c}{ Informative prior } \\
\cline { 4 - 6 } $\boldsymbol{n}$ & & $\boldsymbol{\alpha}$ & Jeffreys' prior & $\boldsymbol{a}_{3}=\boldsymbol{b}_{\mathbf{3}}=\mathbf{4}$ & $\boldsymbol{c}$ \\
\cline { 4 - 6 } 30 & 2.5 & 0.02403470 & 0.00986600 & 0.00847382 & 0.00817383 \\
& 4.0 & 0.52561340 & 0.46056750 & 0.09273483 & 0.85273521 \\
50 & 2.5 & 0.01012250 & 0.00326450 & 0.00278372 & 0.00283672 \\
& 4.0 & 0.32068780 & 0.31650770 & 0.01873292 & 0.01737367 \\
100 & 2.5 & 0.00492160 & 0.00100720 & 0.00097364 & 0.00098215 \\
& 4.0 & 0.11896230 & 0.10766540 & 0.00456722 & 0.00635622 \\
\hline
\end{tabular}




\section{KAUR ET AL}

\section{Full-Bayesian Estimators (Comparison of Symmetrical and Asymmetrical Loss Functions)}

Using the above parameter combinations, mean square errors are computed for Bayesian estimators of parameters of GIWD under the assumptions of squared error loss function (symmetrical), LINEX loss function, and general entropy loss functions (asymmetrical) using Jeffreys' and informative prior.

It is seen from the Tables 8-10 that

(i) Bayesian estimators with informative prior, along with the choice of hyperparameter according to misfit procedure, are found to perform better as compared to Bayesian estimators with Jeffreys' prior because these lead to smaller mean square error for all the estimators for small as well as large sample sizes.

(ii) In the case of informative prior, asymmetrical loss functions (LINEX, entropy loss function) perform better as compared with the symmetrical loss function (SELF) for all the three parameters.

(iii) When sample size increases, mean square error decreases in all cases.

\section{Real Data Examples}

The data of percentage growth of per capita net state domestic product at current prices 2010-11 is taken from Directorate of Economics \& Statistics of respective State Governments, and All-India, CSO as on August 14, 2012. The data fits well to GIWD with $p$-value 0.637 of the Kolmogorov-Smirnov test at $5 \%$ level of significance. The Bayes estimators obtained using semi-Bayesian (GMLE) and full Bayesian approaches have been compared with the classical approach (MLE) to see their relative efficiency. The analysis is done using both informative and noninformative priors and three loss functions, SELF, LINEX loss function, and GELF, along with the same choices of hyperparameters as taken earlier in the case of simulation work. The results obtained are given in Tables 11 and 12 .

Table 11. Mean square error of $\alpha, \beta, \gamma$ (SELF)

\begin{tabular}{rrrrrr} 
& & \multicolumn{2}{c}{ GMLE } & \\
\cline { 3 - 4 } Param. & MLE & Jeffreys' prior & Informative prior & Jeffreys' prior & Informative prior \\
\hline$\alpha$ & 0.0888790000 & 0.0804255000 & 0.8427397000 & 0.0124469000 & 0.0058104080 \\
$\beta$ & 1.8778689000 & 1.8016903500 & 1.9889200000 & 0.0358189800 & $8.78053600 \mathrm{E}-04$ \\
$\gamma$ & 0.0187688000 & 0.0174003300 & 0.0437355000 & 0.0095675800 & 0.0077583277 \\
\hline
\end{tabular}




\section{BAYESIAN ESTIMATION OF GIWD}

Table 12. Mean square error of $\alpha, \beta, \gamma$ (LINEX and GELF)

\begin{tabular}{rrrrr} 
& & & \multicolumn{2}{c}{ Informative prior } \\
\cline { 3 - 5 } Loss function & Parameter & Hyperparameter & $\boldsymbol{b}_{\mathrm{e}}=\mathbf{2}$ & $\boldsymbol{b}_{\mathrm{e}}=\mathbf{- 2}$ \\
\hline GELF & $\alpha$ & $a_{2}=b_{2}=4$ & 0.01034252 & 0.00151386 \\
\hline & & & & \\
\cline { 4 - 5 } & & & Informative prior \\
\cline { 3 - 5 } & & & $\boldsymbol{c}=\mathbf{1}$ & $\boldsymbol{c}=-\mathbf{1}$ \\
\hline LINEX & $\beta$ & $a_{3}=b_{3}=4$ & 0.02345112 & 0.00043727 \\
& $\gamma$ & $a_{1}=b_{1}=6$ & 0.00093425 & 0.00086721 \\
\hline
\end{tabular}

It is seen from Tables 11-12 that

(i) Bayesian estimators with informative prior perform better as compared to Bayesian estimators with Jeffreys' prior.

(ii) In the case of informative prior, asymmetrical loss functions (LINEX, entropy loss function) perform better as compared with the symmetrical loss function (SELF) for all three parameters.

As it is obvious the findings from the analysis of real life example are in accordance with those of simulation study.

\section{Acknowledgements}

The authors are thankful to the anonymous referees and the editor for their valuable suggestions/comments which led to considerable improvement in the manuscript. The authors are also thankful to the UGC and Purse Grant (Panjab University, Chandigarh) for providing financial support for the research work.

\section{References}

Ali, S., Aslam, M., Abbas, N. \& Kazmi, S. M. A. (2012). Scale parameter estimation of the Laplace model using different asymmetric loss functions. International Journal of Statistics and Probability, 1(1), 105-127.

Aslam, M. (2003). An application of prior predictive distribution to elicit the prior density. Journal of Statistical Theory and Applications, 2(1), 70-83. 


\section{KAUR ET AL}

Basu, A. P., \& Ebrahimi, N. (1991). Bayesian approach to life testing and reliability estimation using asymmetric loss function. Journal of Statistical Planning and Inference, 29(1-2), 21-31. doi: 10.1016/0378-3758(92)90118-c

de Gusmão, F. R. S., Ortega, E. M. M., \& Cordeiro, G. M. (2011). The generalized inverse Weibull distribution. Statistical Papers, 52(3), 591-619. doi: 10.1007/s00362-009-0271-3

Drapella, A. (1993). The complementary Weibull distribution: Unknown or just forgotten? Quality and Reliability Engineering International, 9(4), 383-385. doi: 10.1002/qre.4680090426

Guure, C. B., Ibrahimm, N. A., \& Ahmed, A. O. M. (2012). Bayesian estimation of two-parameter Weibull distribution using extension of Jeffreys' prior information with three loss functions. Mathematical Problems in Engineering, 2012, 589640. doi: 10.1155/2012/589640

Jeffreys, H. (1946). An invariant form for the prior probability in estimation problems. Proceedings of the Royal Society A, 186(1007), 453-461. doi: 10.1098/rspa.1946.0056

Jiang, R., Murthy, D. N. P., \& Ji, P. (2001). Models involving two inverse Weibull distributions. Reliability Engineering \& System Safety, 73(1), 73-81. doi: 10.1016/s0951-8320(01)00030-8

Kakwani, N. C. (1980). Income inequality and poverty: Methods of estimation and policy applications. New York, NY: Oxford University Press.

Khan, M. S., Pasha, G.R., \& Pasha, A. H. (2008). Theoretical analysis of inverse Weibull distribution. WSEAS Transactions on Mathematics, 2(7), 30-38.

Lindley, D. V. (1980). Approximate Bayesian methods. In J. M. Bernardo, M. H. De Groot, D. V. Lindley, \& A. F. M. Smith (Eds.), Bayesian statistics (pp. 223-245). Valencia, Spain: University Press.

Lubrano, M. (2014). Lecture 4: Lorenz curves, the Gini coefficient and parametric distributions (unpublished manuscript).

Nash, J. C. (1990). Compact numerical methods for computers: Linear algebra and function minimisation. New York, NY: Hilger.

Nelson, W. B. (1982). Applied life data analysis. New York, NY: John Wiley \& Sons. doi: 10.1002/0471725234

Park, J. S. (2005). A simulation-based hyperparameter selection for quantile estimation of the generalized extreme value distribution. Mathematics and Computers in Simulation, 70(4), 227-234. doi: 10.1016/j.matcom.2005.09.003 


\section{BAYESIAN ESTIMATION OF GIWD}

Parsian, A., Sanjari, F., \& Nematollahi, N. (1993). On the minimaxity of Pitman type estimator under a LINEX loss function. Communications in Statistics - Theory and Methods, 22(1), 97-113. doi: 10.1080/03610929308831008

Raphson, J. (1690). Analysis aequationum universalis [Microfilm]. Ann Arbor, MI: University Microfilms.

Singh, S. K., Singh, U., \& Kumar, D. (2011). Estimation of parameters and reliability function of exponentiated exponential distribution: Bayesian approach under general entropy loss function. Pakistan Journal of Statistics and Operation Research, 7(2), 199-216. doi: 10.18187/pjsor.v7i2.239

Sinha, S. K., \& Howlader, H. A. (1980). On the sampling distributions of Bayesian estimators of the Pareto parameter with proper and improper priors and associated goodness of fit (Technical report No. 103). Manitoba, Canada: University of Manitoba Department of Statistics.

Zaharim, A., Najid, S. K., Razali, A. M., \& Sopian, K. (2009). Analyzing Malaysian wind speed data using statistical distribution. In L. Trilling, D. Perkins, D. D. Dionysiou, L. Perlovsky, K. Davey, D. Landgrebe,... J. W. Lund (Eds.), EE'09 Proceedings of the $4^{\text {th }}$ IASME/WSEAS international conference on energy \& environment (pp. 363-370). Stevens Point, WI: World Scientific and Engineering Academy and Society. 